\title{
Humanitarian Relief Supply Chain Performance Evaluation: A Literature Review
}

\author{
Bethlehem Tamiru Gizaw ${ }^{1} \&$ Alev Taşkin Gümüş ${ }^{1}$ \\ ${ }^{1}$ Department of Industrial Engineering, Yildiz Technical University, Beşiktaş, Istanbul, Turkey \\ Correspondence: Bethlehem Tamiru Gizaw, Department of Industrial Engineering, Yildiz Technical University, \\ Beşiktaş, Istanbul, Turkey. E-mail: butye_lily@yahoo.com
}

Received: January 20, 2016

Accepted: February 10, 2016 Online Published: March 25, 2016

doi:10.5539/ijms.v8n2p105

URL: http://dx.doi.org/10.5539/ijms.v8n2p105

\begin{abstract}
Nowadays small, medium and severe disasters are threatening our world. One of the important role players in alleviating these traits are humanitarian relief supply chains. The increasing number of disasters in our planet earth urges the humanitarian relief supply chains to focus on the assurance of safety of the victims. In order for this to occur, these supply chains should work effectively and efficiently. This can be possible through considerable evaluation of their supply chain performance. In this paper a literature review on supply chain performance evaluation in general and humanitarian relief supply chain performance in particular is presented. Previously conducted researches from the year 2000 until the present time have been reviewed. The works were categorized according to writers, publication year, publishing journal, technique utilized and objective intended. Then an analysis was made on humanitarian relief supply chain literature with respect to the publishing journals and the research technique applied. The result shows that humanitarian relief supply chain evaluation is almost an untouched area which needs further study. Recent supply chain management techniques can be applied for the improved performance of these supply chains. Based on this result, Supply Chain Operations Reference (SCOR), Fuzzy Logic System, and Artificial Neural Networks are found to be the areas which need further study.
\end{abstract}

Keywords: humanitarian relief supply chain, performance evaluation, supply chain

\section{Introduction}

Humanitarian relief works are conducted by different governmental and non-governmental organizations in response to different disasters. Disasters can be calamities, destructive actions, plagues or crises. As a result, these disasters bring small and severe harm to the victims. In order to alleviate the suffering of people, effective and efficient performance of humanitarian relief supply chain is important. This can be achieved through continual evaluation of the performance of the supply chains. Hence, this study reviews the literature on the evaluation of the performance of humanitarian relief supply chains.

\subsection{Supply Chain}

A supply chain encompasses all organizations and activities associated with the flow and transformation of goods from the raw materials stage, through to the end user, as well as the associated information flows. Material and information flows both up and down the supply chain (Handfield \& Nichols, 2013).

\subsection{Humanitarian Relief Supply Chain}

Mentzer et al. (2001) describe the humanitarian supply chain as the network created through the flow of services, supplies, information and finances between donors, beneficiaries, suppliers and different units of humanitarian organizations, in order to provide physical aid to beneficiaries.

Similar to commercial supply chain, supplies flow through the relief chain from the donation to the consumers. There is no single form of humanitarian supply chain, although a typical supply chain could follow the sequence in Figure 1. Government and NGOs are the primary parties involved (Ergun et al., 2009). Governments hold the main power with the control they have over political and economic conditions and directly affect to supply chain processes with their decisions. Donors, public and private organizations are the other significant players in the humanitarian supply chains. Donors have become particularly influential in prompting humanitarian organization to think in terms of greater donor accountability and transparency of the whole supply chain (Wassenhove, 2006). Two-way arrow in the figure represents two-way communications in information, product 
and fund flows among the parties in the humanitarian chain.

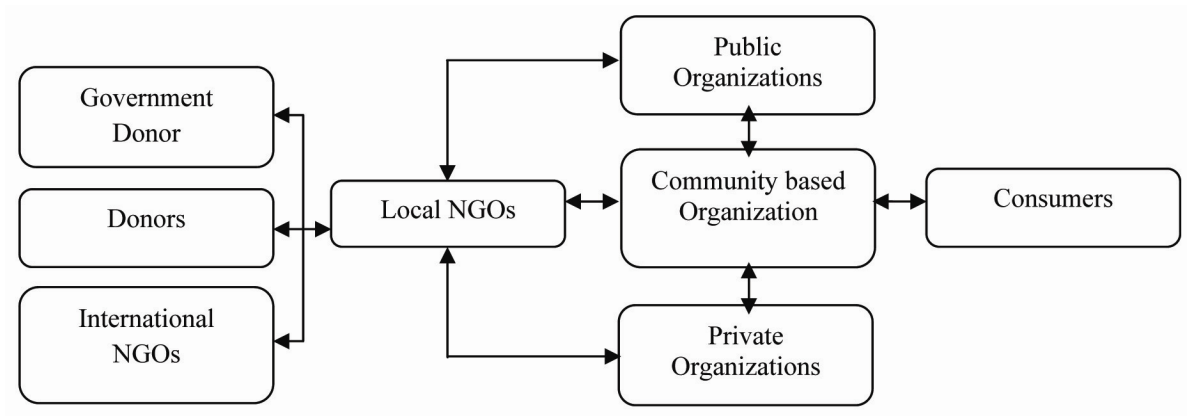

Figure 1. A typical humanitarian supply chain (Chandraprakaikul, W.)

In a disaster context, it is of course important to ensure efficient and effective delivery, such that the appropriate commodities and people reach the victims of the emergency (logistic point of view). However, optimizing the logistic performance requires that all the relationships among the actors involved are managed through an integrated approach to efficiently and effectively coordinate inter-organizational performance, eliminate redundancy, and maximize efficiency along the entire emergency supply chain (supply chain management point of view). In fact, though logistics is more focused on moving something or someone from a point of origin to a destination, supply chain management mainly focuses on relationships among the actors that make such movement possible. Logistics and supply chain management are both crucial to properly set the response to a disaster. Usually, the term "disaster" refers to a "disruption that physically affects a system as a whole and threatens its priorities and goals" (Wassenhove, 2006, p. 476). With respect to cause, it is possible to distinguish between a natural and a man-made disaster; with respect to predictability and speed of occurrence, it is possible to distinguish between a sudden-onset and a slow-onset disaster (Wassenhove, 2006). Taking into account also the different impact in terms of required logistic effort (from higher to lower) it is possible to identify four types of disaster:

- Calamities, characterized by natural causes and sudden-onset occurrences (e.g., earthquakes, hurricanes, tornadoes);

- Destructive actions, characterized by man-made causes and sudden-onsetoccurrences (e.g., terrorist attacks, coups d'état, industrial accidents);

- Plagues, characterized by natural causes and slow-onset occurrence (e.g., famines, droughts, poverty);

- Crises, characterized by man-made causes and slow-onsets occurrence (e.g., political and refugee crises).

Calamities and destructive actions are disasters that demand a higher logistic effort in terms of knowledge and cost because sudden-onset occurrences require a very fast response in devastated areas. The four categories may be interlinked: a calamity (such as an earthquake) may cause plagues (such as an epidemic disease) and crises (such as an economic crisis). Thus, it is sometimes more appropriate to talk about disasters instead of a single disaster.

Different types of disasters need to be managed in different ways: the aid provided to assist in a region's development is distinct from that given to deal with famine and drought; running refugee camps is very different to providing the kind of aid that is needed after a sudden-onset natural disaster or a nuclear accident. Humanitarian efforts are organized along two broad lines (Kovács \& Spens, 2007):

- Disaster relief;

- Continuous aid work.

Ordinarily, disaster relief deals with calamities, destructive actions, and plagues (Long, 1997). Continuous aid work is mainly required in the case of plagues and crises.

Logistics is the most important element in any disaster relief effort, and it is the one that makes the difference between a successful and a failed operation (Wassenhove, 2006). But it is also the most expensive part of any disaster relief: it has been estimated that logistics accounts for about $80 \%$ of the total costs in disaster relief (Wassenhove, V. 2006). And given that the overall annual expenditure of aid agencies is of the order of $\$ 20$ 
billion, the resultant logistic spending is around \$15 billion (Christopher \& Tatham, 2011).Thus, proper investment in logistics in disaster relief provides the main opportunity to develop and implement effective and efficient use of resources in humanitarian operations (Cozzolino et al., 2012). In addition, a more strategic use of resources allows humanitarian organizations to raise donor trust and long-term commitment by increasingly skeptical benefactors (Scholten et al., 2010).

Humanitarian organizations are therefore under greater scrutiny to monitor the impact of aid and the arrangement of their entire operations; they have to prove to donors, who are pledging millions in aid and goods, that they are really reaching the ones in need (Wassenhove V., 2006).

\subsection{Humanitarian Relief Supply Chain Performance}

Humanitarian performance is "the effective collective performance of a complex system of international, national and locally-based organizations, which works to save lives, alleviate suffering and maintain human dignity both during and in the aftermath of man-made crises and natural disasters, as well working to prevent and strengthen preparedness for the occurrence of such situations." In addition "effective performance means undertaking work in ways that are consistent with humanitarian principles, mobilizing and deploying sufficient financial, material and human resources in ways that are relevant, well-managed, accountable, impartial, durable and ensure good quality"(Bölsche, 2013)

\subsection{Humanitarian Supply Chain Performance Evaluation}

Evaluation refers to the process of determining the worth or significance of an activity, policy or program. It is an assessment, as systematic and objective as possible, of a planned, on-going, or completed development intervention. Evaluation in some instances involves the definition of appropriate standards, the examination of performance against those standards, an assessment of actual and expected results and the identification of relevant lessons (OECD/DAC 2002) (ALNAP, 2006).

The evaluation of humanitarian action is "a systematic and impartial examination of humanitarian action intended to draw lessons to improve policy and practice and enhance accountability". Definitions related to humanitarian action tend to stress that evaluations are objective or impartial exercises intended to promote accountability and lesson learning.

Thus supply chain performance evaluation is possible through effective measurement of performances of the supply chain.

An effective performance measurement system:

- Provides the basis to understand the system

- Influences behavior throughout the system

- Provides information regarding the results of system efforts to supply chain members and outside stakeholders

In effect performance measurement is the glue that holds the complex value- creating system together, directing strategic formulation as well as playing a major role in monitoring the implementation of that strategy.

\section{Literature Review}

In this study, previously conducted researches from the year 2000 until the present time have been reviewed. The following section discusses the trend of supply chain performance evaluation in general and humanitarian relief supply chain evaluation in particular.

\subsection{Supply Chain Performance Evaluation in General}

Various techniques have been used to evaluate the performance of supply chains. As it can be observed from the following table, relatively simple methods were used in the early 2000. Then, multi criteria decision making along with managementtechniques began to be applied with an aim to improve supply chain processes.Later a combination of multi criteria decision making tools along with different modeling techniques were used to analyze complicated supply chain processes. 
Table 1. Literature review on humanitarian relief supply chain evaluation

\begin{tabular}{|c|c|c|c|c|}
\hline Writer, Year & Title & Journal Name & Research Technique & Objective \\
\hline $\begin{array}{l}\text { Lai, K. H., Ngai, E. W. T., \& } \\
\text { Cheng, T. C. E. (2003) }\end{array}$ & $\begin{array}{l}\text { An empirical study of supply } \\
\text { chain performance in transport } \\
\text { logistics }\end{array}$ & $\begin{array}{l}\text { International Journal of } \\
\text { Production Economics }\end{array}$ & Cross-sectional survey & $\begin{array}{l}\text { Obtaining a self-assessment of } \\
\text { supply chain performance in } \\
\text { transport logistics by service } \\
\text { providers in the transport } \\
\text { logistics industry }\end{array}$ \\
\hline $\begin{array}{l}\text { Barad, M., \& Sapir, E. D. } \\
(2003)\end{array}$ & $\begin{array}{l}\text { Flexibility in logistic } \\
\text { systems - modeling and } \\
\text { performance evaluation }\end{array}$ & $\begin{array}{l}\text { International Journal of } \\
\text { Production Economics }\end{array}$ & $\begin{array}{l}\text { Trans-routing flexibility and } \\
\text { A multi-factor design of } \\
\text { experiments }\end{array}$ & $\begin{array}{l}\text { Model and evaluate the } \\
\text { performance of the logistic }\end{array}$ \\
\hline $\begin{array}{l}\text { Gunasekarana, A., Patelb, } \\
\text { C., Ronald, E., \& } \\
\text { McGaugheyc (2003) }\end{array}$ & $\begin{array}{l}\text { A framework for supply chain } \\
\text { performance measurement }\end{array}$ & $\begin{array}{l}\text { International Journal of } \\
\text { Production Economics }\end{array}$ & Empirical analysis & $\begin{array}{l}\text { Develop a framework for supply } \\
\text { chain performance measurement } \\
\text { metrics }\end{array}$ \\
\hline Reiner, G. (2004) & $\begin{array}{l}\text { Customer-oriented improvement } \\
\text { and evaluation of supply chain } \\
\text { processes supported by } \\
\text { simulation models }\end{array}$ & $\begin{array}{l}\text { International Journal of } \\
\text { Production Economics }\end{array}$ & $\begin{array}{l}\text { An integrated usage of } \\
\text { discrete-event simulations } \\
\text { models and system } \\
\text { dynamics models }\end{array}$ & $\begin{array}{l}\text { Evaluate and improve the supply } \\
\text { chain processes }\end{array}$ \\
\hline $\begin{array}{l}\text { Wang, F. F., \& Su, C. T. } \\
\text { (2005) }\end{array}$ & $\begin{array}{l}\text { Performance evaluation of a } \\
\text { multi-echelon production, } \\
\text { transportation and distribution } \\
\text { system: A matrix analytical } \\
\text { approach }\end{array}$ & $\begin{array}{l}\text { European Journal of } \\
\text { Operational Research }\end{array}$ & $\begin{array}{l}\text { Matrix analytical computing } \\
\text { approach }\end{array}$ & Evaluate complex supply chains \\
\hline $\begin{array}{l}\text { Yeh, D.Y., Cheng, C. H., \& } \\
\text { Mei-Lin, Ch. (2006) }\end{array}$ & $\begin{array}{l}\text { A modified two-tuple FLC } \\
\text { model for evaluating the } \\
\text { performance of SCM: By the Six } \\
\text { Sigma DMAIC process }\end{array}$ & Applied Soft Computing & $\begin{array}{l}\text { Two-tuple fuzzy linguistic } \\
\text { computing (FLC) }\end{array}$ & $\begin{array}{l}\text { Propose a modified 2-tuple fuzzy } \\
\text { linguistic computing (FLC) } \\
\text { model to evaluate the } \\
\text { performance of Supply } \\
\text { chain management (SCM) }\end{array}$ \\
\hline $\begin{array}{l}\text { Bhagwat, R., \& Sharma, M. } \\
\text { K. (2007) }\end{array}$ & $\begin{array}{l}\text { Performance measurement of } \\
\text { supply chain management: A } \\
\text { balanced scorecard approach }\end{array}$ & $\begin{array}{l}\text { Computers and Industrial } \\
\text { Engineering }\end{array}$ & Balanced Score Card & $\begin{array}{l}\text { Measure and evaluate day-to-day } \\
\text { business operations from } \\
\text { following four perspectives: } \\
\text { finance, customer, internal } \\
\text { business process, and learning } \\
\text { and growth. in small and medium } \\
\text { sized enterprises (SMEs) in India }\end{array}$ \\
\hline $\begin{array}{l}\text { Jammernegg, W., \& Reiner, } \\
\text { G. (2007) }\end{array}$ & $\begin{array}{l}\text { Performance improvement of } \\
\text { supply chain processes by } \\
\text { coordinated inventory and } \\
\text { capacity management }\end{array}$ & $\begin{array}{l}\text { International Journal of } \\
\text { Production Economics }\end{array}$ & $\begin{array}{l}\text { Coordinated inventory and } \\
\text { capacity management and } \\
\text { simulation }\end{array}$ & $\begin{array}{l}\text { Improve the performance of } \\
\text { supply chain processes }\end{array}$ \\
\hline $\begin{array}{l}\text { Chang, I. C., Hwang, H. G., } \\
\text { Liaw, H. C., Hung, M. C., } \\
\text { Chen, S. L., \& Yen, D. C. } \\
\text { (2007) }\end{array}$ & $\begin{array}{l}\text { A neural network evaluation } \\
\text { model for ERP performance } \\
\text { from SCM perspective to } \\
\text { enhance enterprise competitive } \\
\text { advantage }\end{array}$ & $\begin{array}{l}\text { Expert systems with } \\
\text { Applications }\end{array}$ & Neural network & $\begin{array}{l}\text { Evaluate of the performance and } \\
\text { competitive advantages } \\
\text { associated with ERP from a SCM } \\
\text { perspective }\end{array}$ \\
\hline $\begin{array}{l}\text { Yeong-Dong, H., Yi-Ching, } \\
\text { L., \& Jung, L. Jr. (2008) }\end{array}$ & $\begin{array}{l}\text { The performance evaluation of } \\
\text { SCOR sourcing process-the case } \\
\text { study of Taiwan's TFT-LCD } \\
\text { industry }\end{array}$ & $\begin{array}{l}\text { International Journal of } \\
\text { Production Economics }\end{array}$ & Regression analysis & $\begin{array}{l}\text { Evaluate the performance of the } \\
\text { SCOR sourcing processes }\end{array}$ \\
\hline $\begin{array}{l}\text { Tsoulfas, G. T., \& Pappis, C. } \\
\text { P. (2008) }\end{array}$ & $\begin{array}{l}\text { A model for supply chains } \\
\text { environmental performance } \\
\text { analysis and decision making }\end{array}$ & $\begin{array}{l}\text { Journal of Cleaner } \\
\text { Production }\end{array}$ & $\begin{array}{l}\text { Environmental performance } \\
\text { indicators and Multi criteria } \\
\text { decision making }\end{array}$ & $\begin{array}{l}\text { Propose a decision model based } \\
\text { on environmental performance } \\
\text { indicators, which support } \\
\text { decision making in the case of } \\
\text { supply chains in the presence of } \\
\text { environmental considerations }\end{array}$ \\
\hline $\begin{array}{l}\text { Kojima, M., Nakashima, K., } \\
\text { \& Ohno, K. (2008) }\end{array}$ & $\begin{array}{l}\text { Performance evaluation of SCM } \\
\text { in JIT environment }\end{array}$ & $\begin{array}{l}\text { International Journal of } \\
\text { Production Economics }\end{array}$ & $\begin{array}{l}\text { Discrete time } \\
\text { Markov process }\end{array}$ & $\begin{array}{l}\text { Evaluate the performance of the } \\
\text { supply chain management }\end{array}$ \\
\hline $\begin{array}{l}\text { Jiuping, X., Bin, L., \& } \\
\text { Desheng, W. (2009) }\end{array}$ & $\begin{array}{l}\text { Rough data envelopment } \\
\text { analysis and its application to } \\
\text { supply chain performance } \\
\text { evaluation }\end{array}$ & $\begin{array}{l}\text { International Journal of } \\
\text { Production Economics }\end{array}$ & $\begin{array}{l}\text { Rough data envelopment } \\
\text { analysis (RDEA) models }\end{array}$ & $\begin{array}{l}\text { Evaluate the supply chain } \\
\text { network operation efficiency of } \\
\text { the furniture manufacture } \\
\text { industry }\end{array}$ \\
\hline
\end{tabular}




\begin{tabular}{|c|c|c|c|c|}
\hline $\begin{array}{l}\text { Panayides, P. M., \& Lun, Y. } \\
\text { H. V. (2009) }\end{array}$ & $\begin{array}{l}\text { The impact of trust on } \\
\text { innovativeness and supply chain } \\
\text { performance }\end{array}$ & $\begin{array}{l}\text { International Journal of } \\
\text { Production Economics }\end{array}$ & $\begin{array}{l}\text { Hypothesis and structural } \\
\text { equation modeling analysis }\end{array}$ & $\begin{array}{l}\text { Investigate the effects of trust on } \\
\text { innovativeness and supply chain } \\
\text { performance }\end{array}$ \\
\hline $\begin{array}{l}\text { Bayraktar, E., Demirbag, } \\
\text { M., Koh, S. C. L., Tatoglu, } \\
\text { E., \& Zaim, H. (2009) }\end{array}$ & $\begin{array}{l}\text { A causal analysis of the impact } \\
\text { of information systems and } \\
\text { supply chain management } \\
\text { practices on operational } \\
\text { performance: Evidence from } \\
\text { manufacturing SMEs in Turkey }\end{array}$ & $\begin{array}{l}\text { International Journal of } \\
\text { Production Economics }\end{array}$ & $\begin{array}{l}\text { Hypothesis and causal } \\
\text { analysis }\end{array}$ & $\begin{array}{l}\text { Determine the impact of } \\
\text { information systems and supply } \\
\text { chain management on operational } \\
\text { performance }\end{array}$ \\
\hline $\begin{array}{l}\text { Saranga, H., \& Roger, M. R. } \\
\text { (2010) }\end{array}$ & $\begin{array}{l}\text { Performance evaluation of } \\
\text { purchasing and supply } \\
\text { management using value chain } \\
\text { DEA approach }\end{array}$ & $\begin{array}{l}\text { European Journal of } \\
\text { Operational Research }\end{array}$ & $\begin{array}{l}\text { Assessment Survey and the } \\
\text { classical and two-stage } \\
\text { Value Chain Data } \\
\text { Envelopment Analysis } \\
\text { models }\end{array}$ & $\begin{array}{l}\text { develop a comprehensive } \\
\text { performance measurement } \\
\text { framework }\end{array}$ \\
\hline Sun, C. C. (2010) & $\begin{array}{l}\text { A performance evaluation model } \\
\text { by integrating fuzzy AHP and } \\
\text { fuzzy TOPSIS methods }\end{array}$ & $\begin{array}{l}\text { Expert Systems with } \\
\text { Applications }\end{array}$ & $\begin{array}{l}\text { Integrated fuzzy AHP and } \\
\text { fuzzy } \\
\text { TOPSIS methods }\end{array}$ & $\begin{array}{l}\text { Develop a model for performance } \\
\text { evaluation }\end{array}$ \\
\hline $\begin{array}{l}\text { Naini, S. G. J., Aliahmadi, A. } \\
\text { R., \& Jafari-Eskandari, M. } \\
\text { (2010) }\end{array}$ & $\begin{array}{l}\text { Designing a mixed performance } \\
\text { measurement system for } \\
\text { environmental supply chain } \\
\text { management using evolutionary } \\
\text { game theory and balanced score } \\
\text { card: A study of an auto industry } \\
\text { supply chain }\end{array}$ & $\begin{array}{l}\text { Resources, Conservation } \\
\text { and Recycling }\end{array}$ & $\begin{array}{l}\text { Evolutionary game theory } \\
\text { and balanced score card }\end{array}$ & $\begin{array}{l}\text { Develop a framework for a mixed } \\
\text { performance measurement } \\
\text { system for environmental supply } \\
\text { chain management }\end{array}$ \\
\hline $\begin{array}{l}\text { Trkman, P., McCormack, K., } \\
\text { Paulo Valadares de, O. M., } \\
\text { \& Ladeira, M. B. (2010) }\end{array}$ & $\begin{array}{l}\text { The impact of business analytics } \\
\text { on supply chain performance }\end{array}$ & $\begin{array}{l}\text { Decision Support } \\
\text { Systems }\end{array}$ & SCOR and PLS & $\begin{array}{l}\text { Investigate the impact of business } \\
\text { analytics on the performance of } \\
\text { supply chains }\end{array}$ \\
\hline El-Baz, M. A. (2010) & $\begin{array}{l}\text { Fuzzy performance } \\
\text { measurement of a supply chain } \\
\text { in manufacturing companies }\end{array}$ & $\begin{array}{l}\text { Expert Systems with } \\
\text { Applications }\end{array}$ & $\begin{array}{l}\text { Fuzzy set theory and the } \\
\text { pair-wise comparison of } \\
\text { Analytical Hierarchy } \\
\text { Process (AHP) }\end{array}$ & $\begin{array}{l}\text { Develop a framework of supply } \\
\text { chain performance measurement } \\
\text { in manufacturing companies }\end{array}$ \\
\hline Law, Y. W., \& Ngan. (2010) & $\begin{array}{l}\text { Analyzing supply chain } \\
\text { operation models with the } \\
\text { PC-algorithm } \\
\text { and the neural network }\end{array}$ & $\begin{array}{l}\text { Expert Systems with } \\
\text { Applications }\end{array}$ & $\begin{array}{l}\text { PC-Algorithm and neural } \\
\text { network }\end{array}$ & $\begin{array}{l}\text { A two-stage methodology is } \\
\text { applied to an industrial survey } \\
\text { data set to investigate relations } \\
\text { among the key factors in a supply } \\
\text { chain model. }\end{array}$ \\
\hline $\begin{array}{l}\text { Dong, W. C., Young, H. L., } \\
\text { Hwa Ahn, S., \& Min Kyu, } \\
\text { H. (2011) }\end{array}$ & $\begin{array}{l}\text { A framework for measuring the } \\
\text { performance of service supply } \\
\text { chain management }\end{array}$ & $\begin{array}{l}\text { Computers \& Industrial } \\
\text { Engineering }\end{array}$ & $\begin{array}{l}\text { fuzzy analytic hierarchy } \\
\text { process }\end{array}$ & $\begin{array}{l}\text { Development of a framework for } \\
\text { performance measurement of } \\
\text { service supply chain management }\end{array}$ \\
\hline $\begin{array}{l}\text { Gilberto MillerDevo's, G., } \\
\text { Luiz, C., \& Ribeiro, C. } \\
(2011)\end{array}$ & $\begin{array}{l}\text { A fuzzy logic approach to supply } \\
\text { chain performance management }\end{array}$ & $\begin{array}{l}\text { International Journal of } \\
\text { Production Economics }\end{array}$ & $\begin{array}{l}\text { Fuzzy Logic and the metrics } \\
\text { of SCOR model }\end{array}$ & $\begin{array}{l}\text { Performance management of } \\
\text { supply chains }\end{array}$ \\
\hline Lin, R. L. (2011) & $\begin{array}{l}\text { Using fuzzy DEMATEL to } \\
\text { evaluate the green supply chain } \\
\text { management practices }\end{array}$ & $\begin{array}{l}\text { Journal of Cleaner } \\
\text { Production }\end{array}$ & Fuzzy DEMATEL & $\begin{array}{l}\text { Evaluation of the green supply } \\
\text { chain management practices }\end{array}$ \\
\hline $\begin{array}{l}\text { Lin, R. L., Chenb, R. H., \& } \\
\text { Nguyen, T. H. (2011) }\end{array}$ & $\begin{array}{l}\text { Green supply chain management } \\
\text { performance in automobile } \\
\text { manufacturing industry under } \\
\text { uncertainty }\end{array}$ & $\begin{array}{l}\text { Procedia-Social and } \\
\text { Behavioral Sciences }\end{array}$ & $\begin{array}{l}\text { Fuzzy } \\
\text { set theory and Decision } \\
\text { Making Trial and Evaluation } \\
\text { Laboratory }\end{array}$ & $\begin{array}{l}\text { Investigating the performance of } \\
\text { green supply chain management }\end{array}$ \\
\hline $\begin{array}{l}\text { Olugu, E. U., \& Wong, K. Y. } \\
\text { (2011) }\end{array}$ & $\begin{array}{l}\text { An expert fuzzy rule-based } \\
\text { system for closed-loop supply } \\
\text { chain performance assessment in } \\
\text { the automotive industry }\end{array}$ & $\begin{array}{l}\text { Expert Systems with } \\
\text { Applications }\end{array}$ & $\begin{array}{l}\text { Expert fuzzy rule-based } \\
\text { system }\end{array}$ & $\begin{array}{l}\text { Performance evaluation of } \\
\text { closed-loop supply chains }\end{array}$ \\
\hline $\begin{array}{l}\text { Bentes, A. V., Carneiro, J., } \\
\text { Ferreira da, S. J., \& Kimura, } \\
\text { H. (2011) }\end{array}$ & $\begin{array}{l}\text { Multidimensional assessment of } \\
\text { organizational performance: } \\
\text { Integrating BSC and AHP }\end{array}$ & $\begin{array}{l}\text { Journal of Business } \\
\text { Research }\end{array}$ & $\begin{array}{l}\text { Balanced Scorecard (BSC) } \\
\text { and Analytic Hierarchy } \\
\text { Process (AHP) }\end{array}$ & $\begin{array}{l}\text { Organizational performance } \\
\text { assessment }\end{array}$ \\
\hline $\begin{array}{l}\text { Shenc, L., Olfat, L., } \\
\text { Govindan, K., Khodaverdi, } \\
\text { R., \& Diabat, A. (2012) }\end{array}$ & $\begin{array}{l}\text { A fuzzy multi criteria approach } \\
\text { for evaluating green supplier's } \\
\text { performance in green supply } \\
\text { chain with linguistic preferences }\end{array}$ & $\begin{array}{l}\text { Resources, Conservation } \\
\text { and Recycling }\end{array}$ & $\begin{array}{l}\text { A fuzzy multi criteria } \\
\text { approach and fuzzy TOPSIS }\end{array}$ & $\begin{array}{l}\text { Evaluating green supplier's } \\
\text { performance }\end{array}$ \\
\hline
\end{tabular}




\begin{tabular}{|c|c|c|c|c|}
\hline $\begin{array}{l}\text { Qiang, P., \& Nagurney, A. } \\
\text { (2012) }\end{array}$ & $\begin{array}{l}\text { A bi-criteria indicator to assess } \\
\text { supply chain network } \\
\text { performance for critical needs } \\
\text { under capacity and demand } \\
\text { disruptions }\end{array}$ & $\begin{array}{l}\text { Transportation Research } \\
\text { Part A: Policy and } \\
\text { Practice }\end{array}$ & $\begin{array}{l}\text { Bi-criteria performance } \\
\text { indicator }\end{array}$ & $\begin{array}{l}\text { Supply chain network } \\
\text { performance assessment }\end{array}$ \\
\hline $\begin{array}{l}\text { Fan, X., Zhang, S., Wang, } \\
\text { L., Yang, Y., \& Hapeshi, K. } \\
\text { (2013) }\end{array}$ & $\begin{array}{l}\text { An Evaluation Model of Supply } \\
\text { Chain Performances Using } \\
\text { 5DBSC and LMBP Neural } \\
\text { Network Algorithm }\end{array}$ & $\begin{array}{l}\text { Journal of Bionic } \\
\text { Engineering }\end{array}$ & $\begin{array}{l}5 \text { Dimensional Balanced } \\
\text { Scorecard (5DBSC) and } \\
\text { LMBP } \\
\text { (Levenberg-Marquardt Back } \\
\text { Propagation) } \\
\text { neural network }\end{array}$ & $\begin{array}{l}\text { Performance evaluation of supply } \\
\text { chains }\end{array}$ \\
\hline $\begin{array}{l}\text { Mansoornejada, B., } \\
\text { Pistikopoulosb, E. N., \& } \\
\text { Stuart, P. (2013) }\end{array}$ & $\begin{array}{l}\text { Metrics for evaluating the forest } \\
\text { bio refinery supply chain } \\
\text { performance }\end{array}$ & $\begin{array}{l}\text { Computers and Chemical } \\
\text { Engineering }\end{array}$ & $\begin{array}{l}\text { Mixed integer linear } \\
\text { programming (MILP) }\end{array}$ & $\begin{array}{l}\text { Developing supply chain } \\
\text { performance evaluation metrics }\end{array}$ \\
\hline $\begin{array}{l}\text { Vidalis, M. I., } \\
\text { Koukoumialos, S., \& } \\
\text { Michael, G. (2013) }\end{array}$ & $\begin{array}{l}\text { Performance evaluation of a } \\
\text { merge supply network: A } \\
\text { distribution center with multiple } \\
\text { reliable random suppliers }\end{array}$ & $\begin{array}{l}\text { Computers and Industrial } \\
\text { Engineering }\end{array}$ & $\begin{array}{l}\text { Markov process modeling } \\
\text { and computational algorithm } \\
\text { development }\end{array}$ & $\begin{array}{l}\text { Modeling performance } \\
\text { evaluation of merge supply } \\
\text { network }\end{array}$ \\
\hline $\begin{array}{l}\text { Bourlakis, M., Maglaras, G., } \\
\text { Aktas, E., Gallear, D., \& } \\
\text { Fotopoulos, C. (2013) }\end{array}$ & $\begin{array}{l}\text { Firm size and sustainable } \\
\text { performance in food supply } \\
\text { chains: Insights from Greek } \\
\text { SMEs }\end{array}$ & $\begin{array}{l}\text { International Journal of } \\
\text { Production Economics }\end{array}$ & $\begin{array}{l}\text { Sustainable performance } \\
\text { measures and statistical } \\
\text { analysis }\end{array}$ & $\begin{array}{l}\text { Assessing sustainable } \\
\text { performance of food supply } \\
\text { chains }\end{array}$ \\
\hline $\begin{array}{l}\text { Shafiee, M., Hosseinzadeh, } \\
\text { L. F., \& Hilda, S. H. (2014) }\end{array}$ & $\begin{array}{l}\text { Supply chain performance } \\
\text { evaluation with data } \\
\text { envelopment analysis and } \\
\text { balanced scorecard approach }\end{array}$ & $\begin{array}{l}\text { Applied Mathematical } \\
\text { Modeling }\end{array}$ & $\begin{array}{l}\text { DEMATEL approach, } \\
\text { balanced scorecard (BSC) } \\
\text { and data envelopment } \\
\text { analysis (DEA) model }\end{array}$ & $\begin{array}{l}\text { Performance evaluation of supply } \\
\text { chains }\end{array}$ \\
\hline $\begin{array}{l}\text { Chithambaranathan, P., } \\
\text { Subramanian, N., } \\
\text { Gunasekaran, A., \& } \\
\text { Palaniappan, P. L. K. (2015) }\end{array}$ & $\begin{array}{l}\text { Service supply chain } \\
\text { environmental performance } \\
\text { evaluation using grey based } \\
\text { hybrid MCDM approach }\end{array}$ & $\begin{array}{l}\text { International Journal of } \\
\text { Production Economics }\end{array}$ & $\begin{array}{l}\text { Grey based method with } \\
\text { ELECTRE and VIKOR } \\
\text { approaches }\end{array}$ & $\begin{array}{l}\text { Service supply chain green } \\
\text { performance evaluation }\end{array}$ \\
\hline $\begin{array}{l}\text { Bukhori, I. B., Widodo, K. } \\
\text { H., \& DyahIsmoyowati. } \\
(2015)\end{array}$ & $\begin{array}{l}\text { Evaluation of Poultry Supply } \\
\text { Chain Performance in XYZ } \\
\text { Slaughtering House Yogyakarta } \\
\text { using SCOR and AHP Method }\end{array}$ & $\begin{array}{l}\text { Agriculture and } \\
\text { Agricultural Science } \\
\text { Procedia }\end{array}$ & $\begin{array}{l}\text { SCOR, AHP and cause- } \\
\text { and-effect diagram }\end{array}$ & $\begin{array}{l}\text { Evaluating Poultry Supply Chain } \\
\text { Performance }\end{array}$ \\
\hline $\begin{array}{l}\text { Rostamzadeh, R., Govindan, } \\
\text { K., Esmaeili, A., \& Sabaghi, } \\
\text { M.(2015) }\end{array}$ & $\begin{array}{l}\text { Application of fuzzy VIKOR for } \\
\text { evaluation of green supply chain } \\
\text { management practices }\end{array}$ & Ecological Indicators & Fuzzy VIKOR & $\begin{array}{l}\text { Evaluating green supply chain } \\
\text { management practices }\end{array}$ \\
\hline
\end{tabular}

\subsection{Humanitarian Relief Performance Evaluation in Particular}

According to (Wassenhove, 2006), in 2006, humanitarian organizations were about 15 years behind the private sector in terms of understanding the importance of using efficient supply chains and opportunities to 'go global'. Wassenhove (2006) also states that it is only recently that humanitarian organization such as Red Cross and the World Food Programme (WFP) have started to pin-point logistics and supply chain management as key to a relief organization.

The Active Learning Network for Accountability and Performance in Humanitarian Action has worked out four central requirements for performance measurement in Humanitarian Action: efficiency, effectiveness, impact, sustainability, relevance, appropriateness, connectedness, coherence and coverage.

Studies with an objective of identifying the challenges of humanitarian supply chain have been carried out. Literature reviews were also done to understand the performance challenges and to foresight the future areas of research. To measure the performance of humanitarian supply chain different researches were conducted accompanied with case studies.

Wider concepts such as balanced score card was also used as a performance measuring tool to study humanitarian supply chains with the perspectives of customer, internal process, learning and growth, and finance. In a similar manner, a process oriented approach using SCOR model was implemented to make use of the three components of process, performance, and best practices integrating organizations from different sectors.

In recent years few researches applied multi criteria decision making tools for analysis and evaluation of humanitarian supply chains. This shows that a lot of studies need to be carried out to make use of recent 
techniques of supply chain performance evaluation.

Table 2. Humanitarian relief supply chain performance evaluation

\begin{tabular}{|c|c|c|c|c|}
\hline Writer, Year & Title & Journal Name & Research \& Technique & Objective \\
\hline Rodman, W. K. & $\begin{array}{l}\text { Supply chain management in } \\
\text { humanitarian relief logistics }\end{array}$ & $\begin{array}{l}\text { Thesis (Air Force Institute } \\
\text { Of Technology } \\
\text { Wright-Patterson Air } \\
\text { Force Base, Ohio) }\end{array}$ & $\begin{array}{l}\text { Grounded theory and supply } \\
\text { chain barriers were analyzed } \\
\text { based on academic, } \\
\text { organizational, and } \\
\text { contemporary literature } \\
\end{array}$ & $\begin{array}{l}\text { To explore the use of supply chain } \\
\text { management techniques to } \\
\text { overcome barriers encountered by } \\
\text { logistics managers during } \\
\text { humanitarian relief operations }\end{array}$ \\
\hline $\begin{array}{l}\text { Beamon, B. M., \& } \\
\text { Burcu, B. (2008) }\end{array}$ & $\begin{array}{l}\text { Performance measurement in } \\
\text { humanitarian relief chains }\end{array}$ & $\begin{array}{l}\text { International Journal of } \\
\text { Public Sector } \\
\text { Management }\end{array}$ & $\begin{array}{l}\text { Interviews with extended } \\
\text { analysis using a case study }\end{array}$ & $\begin{array}{l}\text { Present a framework that can be } \\
\text { used as a basis for a performance } \\
\text { measurement system in the relief } \\
\text { sector }\end{array}$ \\
\hline Jussi, H. J. & $\begin{array}{l}\text { Global supply chain management } \\
\text { and performance measurement } \\
\text { Literature Review }\end{array}$ & $\begin{array}{l}\text { Leka Project (Savonia } \\
\text { University of Applied } \\
\text { Sciences and Tampere } \\
\text { University of Technology) } \\
\end{array}$ & Literature Review & $\begin{array}{l}\text { Reviewing literature on supply } \\
\text { chain management and performance } \\
\text { measurement }\end{array}$ \\
\hline $\begin{array}{l}\text { Chandraprakaikul, } \\
\text { W. }\end{array}$ & $\begin{array}{l}\text { Humanitarian supply chain } \\
\text { management: Literature review } \\
\text { and future research }\end{array}$ & $\begin{array}{l}\text { University of the Thai } \\
\text { Bangkok }\end{array}$ & Literature Review & $\begin{array}{l}\text { To identify trends on the existing } \\
\text { literature and carry out analysis to } \\
\text { establish areas for future research }\end{array}$ \\
\hline Bölsche, D. & $\begin{array}{l}\text { Performance Measurement in } \\
\text { Humanitarian Logistics - a } \\
\text { process-oriented perspective }\end{array}$ & $\begin{array}{l}\text { University of Applied } \\
\text { Sciences Fulda, Germany }\end{array}$ & SCOR & $\begin{array}{l}\text { To improve the effectiveness and } \\
\text { efficiency of humanitarian supply } \\
\text { chain by a process approach through } \\
\text { the application of SCOR model. }\end{array}$ \\
\hline $\begin{array}{l}\text { Abid, H., \& } \\
\text { Klumpp, M. }\end{array}$ & $\begin{array}{l}\text { Performance measurement in } \\
\text { Humanitarian logistics: a } \\
\text { literature review }\end{array}$ & $\begin{array}{l}\text { FOM university of } \\
\text { Applied sciences }\end{array}$ & Literature Review & $\begin{array}{l}\text { To provide a literature review and } \\
\text { outline future research } \\
\text { opportunities in performance } \\
\text { measurement in humanitarian } \\
\text { logistics }\end{array}$ \\
\hline $\begin{array}{l}\text { Zafeiridis, D. W. S. } \\
(2013)\end{array}$ & $\begin{array}{l}\text { Challenges and the use of } \\
\text { performance measurements in } \\
\text { humanitarian supply chains }\end{array}$ & $\begin{array}{l}\text { Master's thesis } \\
\text { (Jönköping Universsity) }\end{array}$ & A holistic multiple case study & $\begin{array}{l}\text { To identify the main challenges in } \\
\text { humanitarian supply chains and the } \\
\text { role of performance measurements } \\
\text { in humanitarian operations thus } \\
\text { identifying an appropriate model for } \\
\text { measurement }\end{array}$ \\
\hline $\begin{array}{l}\text { Abidi, H., Leeuw, } \\
\text { S. D., \& Klumpp, } \\
\text { M. (2013) }\end{array}$ & $\begin{array}{l}\text { Measuring success in } \\
\text { humanitarian supply chains }\end{array}$ & $\begin{array}{l}\text { International Journal of } \\
\text { Business and } \\
\text { Management Invention }\end{array}$ & Case study approach & $\begin{array}{l}\text { To define success factors and } \\
\text { translate them into concrete } \\
\text { indicators supported by a case study }\end{array}$ \\
\hline $\begin{array}{l}\text { Santarelli, G., Abidi, } \\
\text { H., Regattieri, A., \& } \\
\text { Klumpp, M. }\end{array}$ & $\begin{array}{l}\text { A performance measurement } \\
\text { system for the evaluation of } \\
\text { humanitarian supply chains }\end{array}$ & $\begin{array}{l}\text { University of Padua, } \\
\text { FOM University and } \\
\text { Amsterdam University }\end{array}$ & $\begin{array}{l}\text { Categorized key performance } \\
\text { indicators with Case Studies }\end{array}$ & $\begin{array}{l}\text { To measure the performance of } \\
\text { humanitarian supply chains during } \\
\text { both disaster situations and } \\
\text { development }\end{array}$ \\
\hline $\begin{array}{l}\text { Erkan, C. E., } \\
\text { Gumus, A. T., \& } \\
\text { Alegoz, M. (2014) }\end{array}$ & $\begin{array}{l}\text { A trapezoidal type- } 2 \text { fuzzy } \\
\text { MCDM method toidentify and } \\
\text { evaluate critical success } \\
\text { factorsfor humanitarian relief } \\
\text { logistics management }\end{array}$ & $\begin{array}{l}\text { Journal of Intelligent and } \\
\text { Fuzzy Systems: } \\
\text { Applications in } \\
\text { Engineering and } \\
\text { Technology }\end{array}$ & $\begin{array}{l}\text { Trapezoidal interval type- } 2 \\
\text { fuzzy AHP method }\end{array}$ & $\begin{array}{l}\text { Identifying and evaluating the } \\
\text { success factors which are critical for } \\
\text { effective management of } \\
\text { humanitarian relief logistics } \\
\text { operations }\end{array}$ \\
\hline Leeuw, S. D. (2014) & $\begin{array}{l}\text { Towards a reference mission map } \\
\text { for performance measurement in } \\
\text { humanitarian supply chains }\end{array}$ & $\begin{array}{l}\text { Collaborative Networks } \\
\text { for a Sustainable World }\end{array}$ & $\begin{array}{l}\text { Literature and four mini-case } \\
\text { studies using Balanced Score } \\
\text { Card }\end{array}$ & $\begin{array}{l}\text { To develop a reference mission map } \\
\text { for a humanitarian supply chain. }\end{array}$ \\
\hline Haavisto, I. (2014) & $\begin{array}{l}\text { Performance in humanitarian } \\
\text { supply chains }\end{array}$ & $\begin{array}{l}\text { Hanken school of } \\
\text { economics }\end{array}$ & $\begin{array}{l}\text { Contingency and game setting } \\
\text { theory }\end{array}$ & $\begin{array}{l}\text { To analyze how supply chain } \\
\text { performance is understood in the } \\
\text { humanitarian context }\end{array}$ \\
\hline
\end{tabular}

\section{Discussion}

Different journals have published papers related to supply chain performance evaluation. International Journal of Production Economics has the highest share of papers (13; 34.2\%), then Expert Systems with Applications (5; $13.16 \%)$, Computers and Industrial Engineering (3; 7.89\%), Resources Conservation and Recycling, Journal of 
Cleaner Production and European Journal of Operational Research (2; 5.26\%), Ecological Indicators; Agriculture and Agricultural Science Procedia; Journal of Intelligent \& Fuzzy Systems: Applications in Engineering and Technology; Applied Mathematical Modeling; Computers and Chemical Engineering; Journal of Bionic Engineering; Transportation Research Part A: Policy and Practice; Journal of Business Research; Procedia - Social and Behavioral Sciences; Decision Support Systems and Applied Soft Computing $(1 ; 2.63 \%)$. It can be shown in the following figure as shown below:

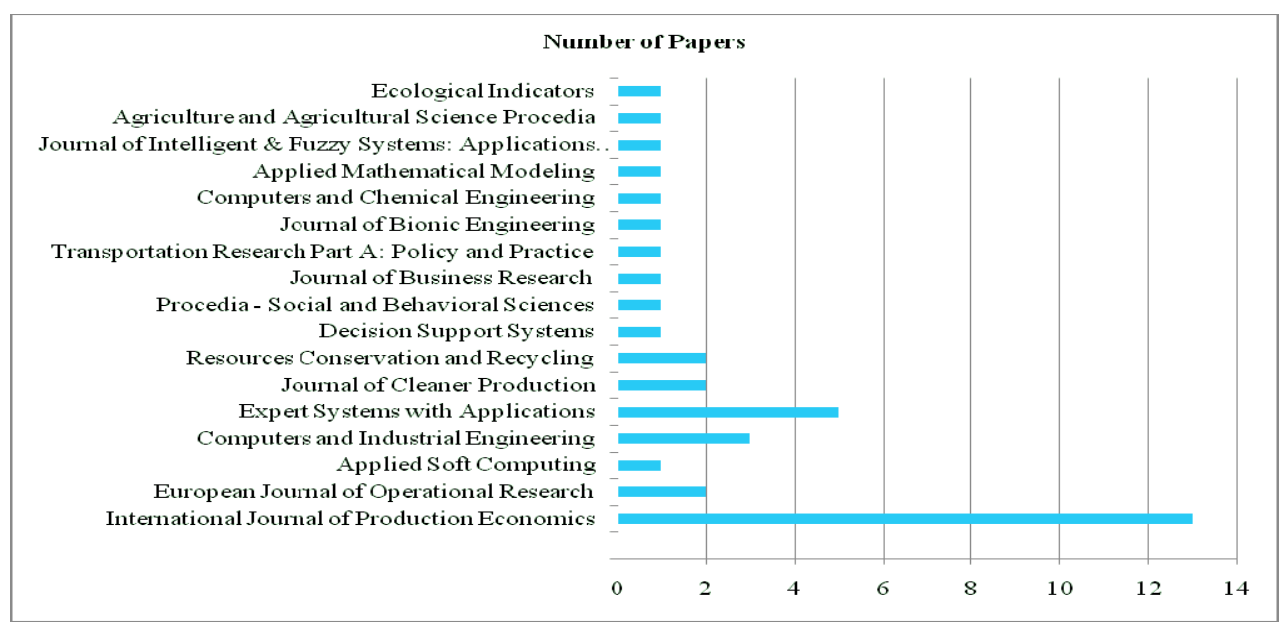

Figure 2. Number of papers published in different journals

The number of papers that are directly related with supply chain performance evaluation grouped under the different techniques utilized presented in the figure below. Fuzzy logic combined with multi-criteria decision making; empirical analysis and multi criteria decision making have the highest application $(8 ; 21.05 \%)$, followed by SCOR and multi criteria decision making (4; 10.5\%), Balanced Score Card and multi criteria decision making (3; 7.89\%); simulation (2; 5.26\%); Balanced Score Card, SCOR and fuzzy logic, neural network, neural network and multi criteria decision making and Balanced Score Card and neural network $(1 ; 2.63 \%)$

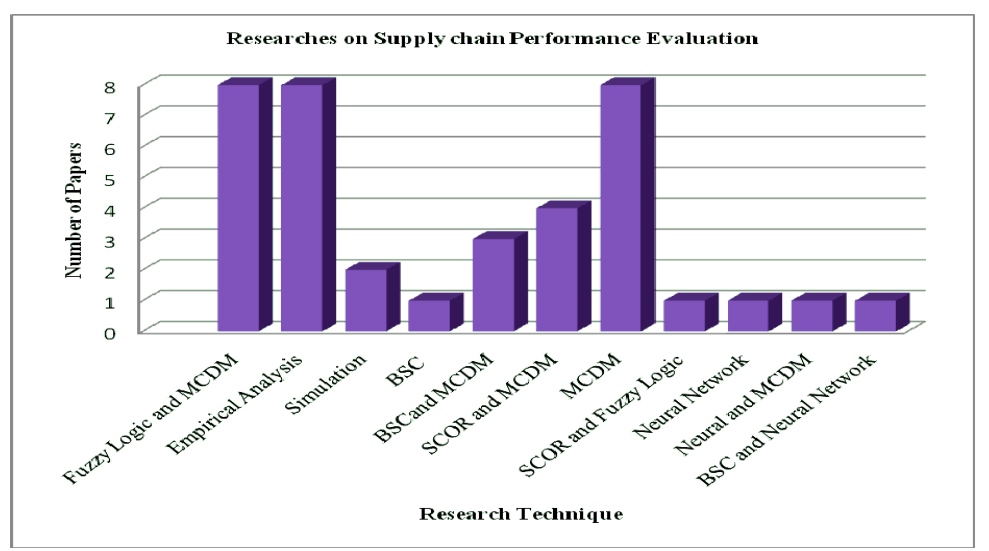

Figure 3. Research techniques used for supply chain performance evaluation

Note. $\mathrm{MCDM}=$ Multi Criteria Decision Making; $\mathrm{BSC}=$ Balanced Score Card; SCOR= Supply Chain Operations Reference.

When we come to the analysis of humanitarian relief supply chain performance in particular, as can be seen from Table 2, enough research has not been made that can lead us to a statistical analysis as in the condition of supply chain performance evaluation in particular. 
The increasing number of disasters in our planet earth urges the humanitarian relief supply chains to focus on the assurance of safety of the victims. This can be possible through efficient evaluation of their supply chain performance.

There are different models of measuring the performance of supply chains. ABC: Activity-Based Costing, FLR: Framework for Logistics Research, BSC: Balanced ScoreCard, SCOR: Supply Chain Operation Reference model, GSCF framework, ASLOG audit, SASC: Strategic Audit Supply Chain, Global EVALOG (Global MMOG/LE), WCL: World Class Logistics model, AFNOR FD X50-605, SCM/SME, APICS, ECR: Efficient Customer Response, EFQM: Excellence model, SCALE: Supply Chain Advisor Level Evaluation and SPM: Strategic Profit Model can be mentioned. Among these models,

Among the different supply chain evaluation moding techniques SCOR: Supply Chain Operation Reference model is a rigorous and proven methodology for systematic supply chain performance improvement. It offers a step by step engineering approach that can help to analyze, design and improve supply chain performance. It combines elements of business process reengineering metrics, bench marking and leading practices in to a single framework.

\subsection{Supply-Chain Operations Reference (SCOR)}

The Supply-Chain Operations Reference (SCOR) model was developed by the Supply-Chain Council (SCC) to assist firms in increasing the effectiveness of their supply chains, and to provide a process-based approach to Supply Chain Management. (Lockamy III \& McCormack, 2004)

SCOR is a process reference model which combines the concepts of business process reengineering, benchmarking and best practices.

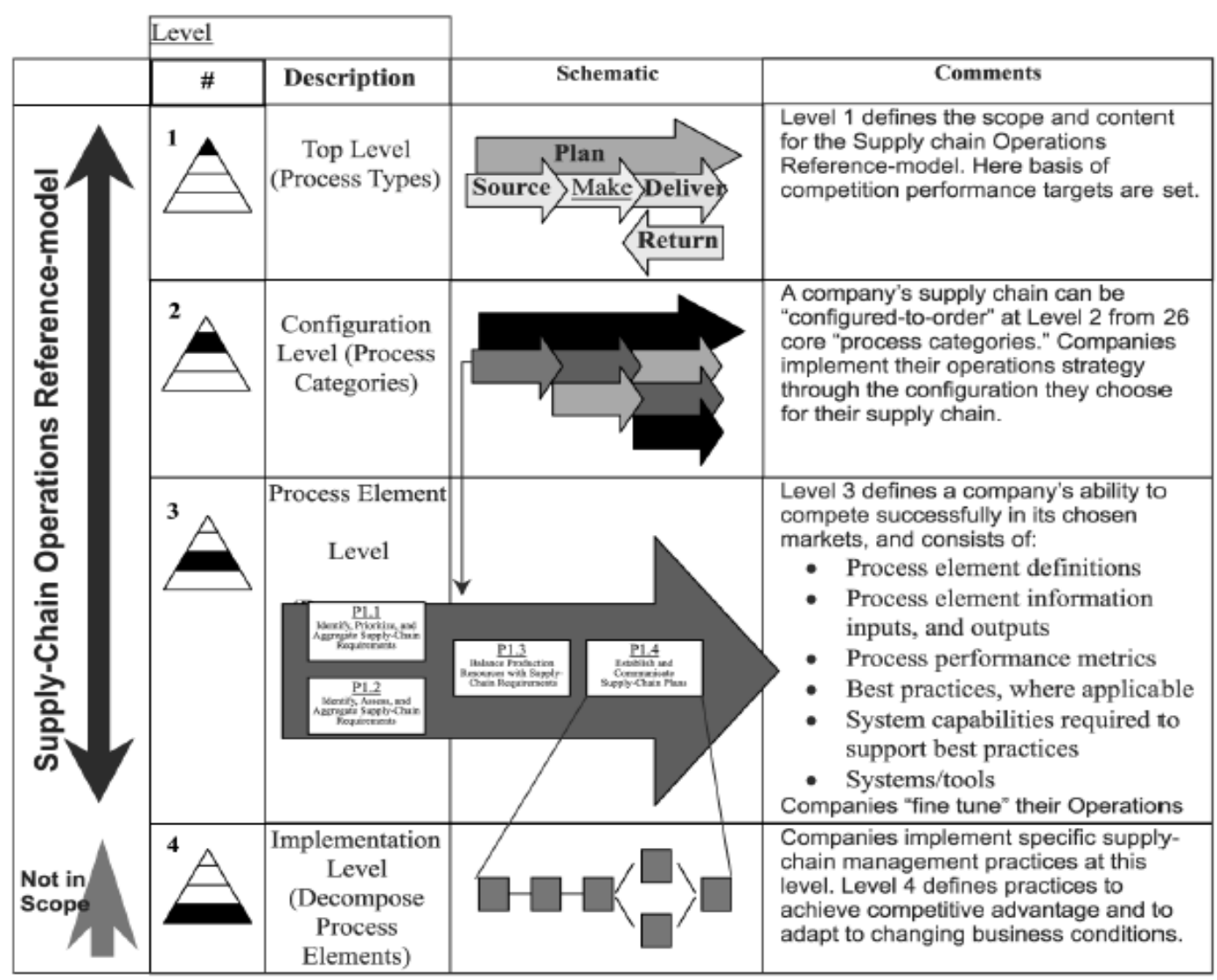

Figure 4. Hierarchical model of SCOR with specific boundaries in regard to scope (Lockamy \& McCormack, 2004)

Besides the necessity of efficiency, supply chains perform an adverse competitive environment where the future is uncertain and under the condition where performance metrics need to be evaluated. This scenario made SCOR 
implementation along with along Fuzzy Logic and Artificial Neural Network (ANN) more important. They both are interactive software based systems used to support business and organizational decision making activities.

\subsection{Fuzzy Logic System}

A fuzzy logic system is unique in that it is able to simultaneously handle numerical data and linguistic knowledge. It is a nonlinear mapping of an input data (feature) vector into a scalar output, i.e. it maps numbers into numbers. Fuzzy set theory and fuzzy logic establish the specifics of the nonlinear mapping.

In general, a fuzzy logistics system is a nonlinear mapping of an input data (feature) vector into a scalar output (the vector output case decomposes into a collection of independent multi input/single-output systems). The richness of fuzzy logistics is that there are enormous numbers of possibilities that lead to lots of different mappings.

Principle of Incompatibility is quoted in different papers, which many take as a rationale for the study of FL in engineering and other disciplines: "As the complexity of a system increases, our ability to make precise and yet significant statements about its behavior diminishes until a threshold is reached beyond which precision and significance (or relevance) become almost mutually exclusive characteristics" or, "The closer one looks at a real-world problem, the fuzzier becomes its solution."

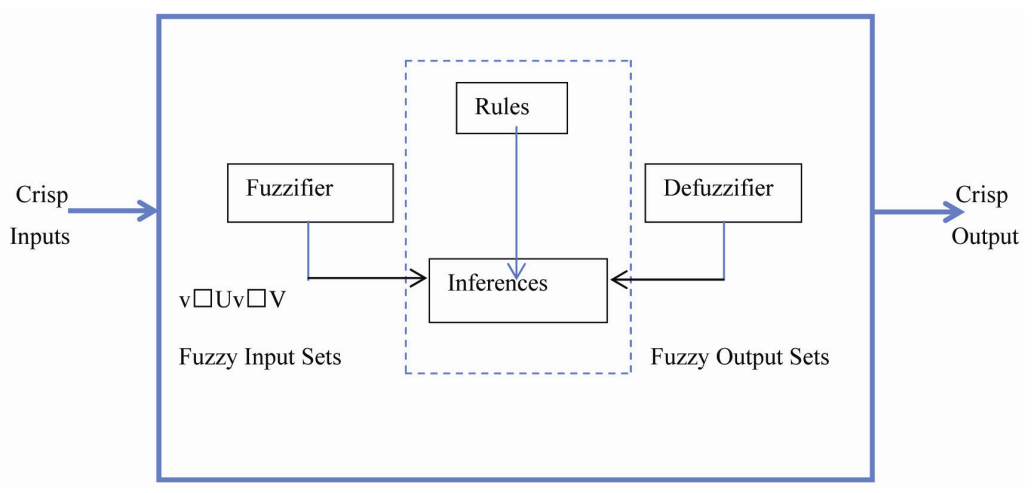

Figure5. A fuzzy logic system

Figure 5 depicts a FLS that is widely used in fuzzy logic controllers and signal processing applications. A FLSmaps crisp inputs into crisp outputs. It contains four components: rules, fuzzifier, inference engine, and defuzzifier. Once the rules have been established, a FLS can be viewed as a mapping from inputs to outputs (the solid path in Figure 5, from "Crisp Inputs" to "Crisp Outputs"), and this mapping can be expressed quantitatively as $y=f(z)$. Rulesmay be provided by experts (you may be such a person) or can be extracted from numerical data. In either case, engineering rulesare expressed as a collection of IFTHEN statements, e.g., "IF u1 is very warm and $1 \sim 2$ is quite low, THEN turn it somewhat to the right." This one rule reveals that we will need an understanding of: 1) linguistic variables versus numerical values of a variable (e.g., very warm versus $36^{\circ} \mathrm{C}$ ); 2 ) quantifying linguistic variables (e.g., with it, ranging from extremely hot to extremely cold), which is done using fuzzy membership functions; 3) logical connections for linguistic variables (e.g., "and," "or," etc.); and 4) implications, i.e., "IF A THEN B." Additionally, we will need to understand how to combine more than one rule. The fuzzifier maps crisp numbers into fuzzy sets. It is needed in order to activate rules which are in terms of linguistic variables, which have fuzzy sets associated with them.

The inference engine of the FLS maps fuzzy sets into fuzzy sets. It handles the way in which rules are combined. Just as we humans use many different types of inferential procedures to help us understand things or to make decisions, there are many different fuzzy logic inferential procedures. Only a very small number of them are actually being used in engineering applications of FL.

In many applications, crisp numbers must be obtained at the output of a FLS. The defuzzifier maps output sets into crisp numbers. In a controls application, for example, such a number corresponds to a control action. In a signal processing application, such a number could correspond to the prediction of next year's sunspot activity, a financial forecast, or the location of a target (Zadeh, 1965). 


\subsection{Artificial Neural Network}

An Artificial Neural Network (ANN) is a mathematical model that tries to simulate the structure and functionalities of biological neural networks. Basic building block of every artificial neural network is artificial neuron, that is, a simple mathematical model (function). Such a model has three simple sets of rules: multiplication, summation and activation.

Atthe entrance of artificial neuron the inputs are weighted what means that every input valueis multiplied with individual weight. In the middle section of artificial neuron is sum function that sums all weighted inputs and bias. At the exit of artificial neuron the sum of previously weighted inputs and bias is passing through activation function that is also called transfer function. Transfer function defines the properties of artificial neuron and can be any mathematical function. We choose it on the basis of problem that artificial neuron (artificial neural network) needs to solve and in most cases we choose it from the following set of functions: Step function, Linear function and Non-linear (Sigmoid) function.

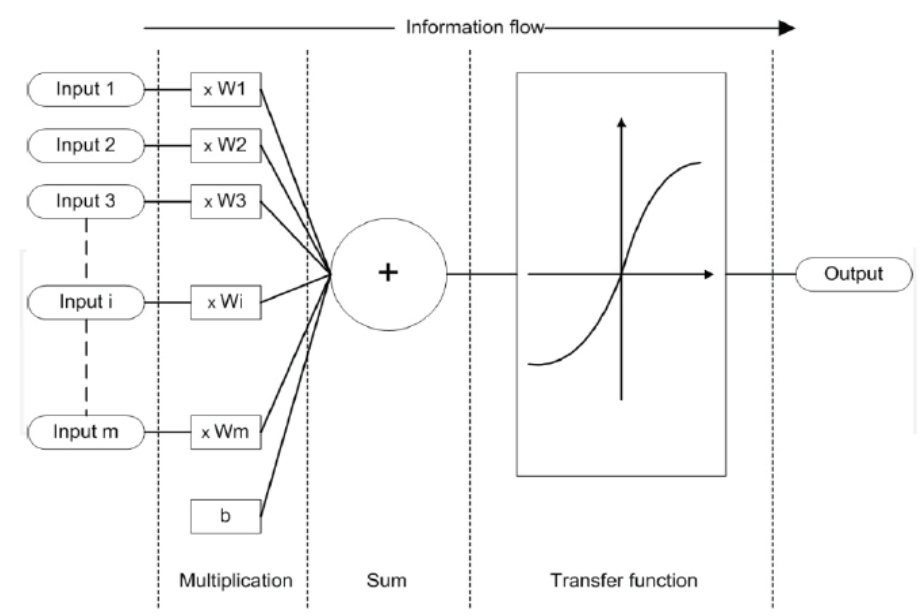

Figure 6. Working principle of an artificial neuron (Krenker A., Bešter J., \& Kos A., 2013)

\section{Conclusion}

Humanitarian relief supply chain evaluation is almost the untouched area with application with software based systems like the previously mentioned ones. As human life is the main component humanitarian relief supply chain, the application of SCOR model with the application of Fuzzy logic and Artificial Neural Network (ANN) would bring great benefit to the state of the art and also functions as an initiator of researchers on this research area.

\section{References}

Abdi, H., Leeuw, S. D., \& Klumpp, M. (2013). Measuring success in humanitarian supply chains.

Abidi, H., \& Klumpp, M. (n.d.). Performance measurement in Humanitarian logistics: a literature review.

Agrell, P. J., \& Marbini, A. H. (2013). Frontier-based performance analysis models for supply chain management: state of the art and research directions. Computers and Industrial Engineering. http://dx.doi.org/10.1016/j.cie.2013.02.014

Al Shamisi, M. H., Assi, A. H., \& Hejase, H. A. N. (2010). Using MATLAB to Develop Artificial Neural Network Models for Predicting Global Solar Radiation in Al Ain City - UAE United Arab Emirates University, United Arab Emirates. Retrieved from www.intechopen.com

ALNAP. (2006). Evaluating humanitarian action using the OECD-DAC criteria-an ALNAP guide for humanitarian agencies. ALNAP, London.

ALNAP: Ver.130. Learning, Accountability Performance in Humanitarian Action. Retrieved from http://www.alnap.org

APICS dictionary. (2013). Supply chain management. Retrieved from http://www.apics.org/dictionary/dictionary-information?ID=4202 
Asian Development Bank. (2004). Disaster and Emergency Assistance Policy.

Australian Council for International Development's Humanitarian Reference Group [ACFID]. (2007). Emergency response supply chain assessment.

Awan, Z., \& Rahman, Z. (2010). Supply Chain Designs for Humanitarian Relief. Jönköping University, Jönköping.

Barad, M., \& Sapir, D. E. (2003). Flexibility in logistic systems-modeling and performance evaluation.

Bayraktar, E., Demirbag, M. S. C., Koh L., Tatoglu, E., \& Zaim, H. (2009). A causal analysis of the impact of information systems and supply chain management practices on operational performance: Evidence from manufacturing SMEs in Turkey.

Beamon, B. M., \& Balcik, B. (2005). Distribution Network Design for Humanitarian Relief Chains. IE Graduate Seminar, November, 8. University of Washington, Industrial Engineering.

Beamon, B. M., \& Balcik, B. (2008). Distribution Network Design for Humanitarian Relief Chains. IE Graduate Seminar, 21(1). University of Washington.

Beamon, B. M., \& Balcik, B. (2008). Performance measurement in humanitarian relief chains. International Journal of Public Sector Management, 21(1), 4-25. http://dx.doi.org/10.1108/09513550810846087

Bentes, A. V., Carneiro, J., Ferreira, da S. J., \& Kimura, H. (2011). Multidimensional assessment of organizational performance: Integrating BSC and AHP.

Bhagwat, R., \& Sharma, M. K. (2007). Performance measurement of supply chain management: A balanced scorecard approach.

Bölsche, D. (2013). Performance measurement in humanitarian logistics. Discussion Papers in Business and Economics. Fulda University of Applied Sciences, Fulda.

Bourlakis, M., Maglaras, G., Aktas, E., Gallear, D., \& Fotopoulos, C. (2013). Firm size and sustainable performance in food supply chains: Insights from Greek SMEs.

Bui, T., Cho, S., Sankaran, S., \& Sovereign, M. (2000). A framework for designing a global information network for multinational humanitarian assistance/disaster relief. Information Systems Frontiers, 1(4), 427-442. http://dx.doi.org/10.1023/A:1010074210709

Bukhori, I. B., Widodo, K. H., \& Ismoyowati, D. (2015). Evaluation of Poultry Supply Chain Performance in XYZ Slaughtering House Yogyakarta using SCOR and AHP Method.

Celik, E., Gumus, A. T., \& Alegoz, M. (2014). A trapezoidal type-2 fuzzy MCDM method to identify and evaluate critical success factors for humanitarian relief logistics management.

Chandraprakaikul, W. (2010). A guiding framework for designing humanitarian relief supply chains-A case study inThailand. POMS 21st Annual Conference, Vancouver, Canada.

Chandraprakaikul, W. (n.d.). Humanitarian Supply Chain Management: Literature Review and Future Research.

Charles, A., Lauras, M., \& Van Wassenhove, L. N. (2010). A model to define and assess the agility of supply chains: Building on humanitarian experience. International Journal of Physical Distribution \& Logistics.

Chia. C. S. (2010). A performance evaluation model by integrating fuzzy AHP and fuzzy TOPSIS methods.

Chithambaranathan, P., Nachiappan, S., Angappa, G., \& Palaniappan, P. L. K. (2015). ServiceSupply Chain Environmental Performance Evaluation using Grey based hybrid MCDM approach. International Journal of Production Economics. http://dx.doi.org/10.1016/j.jpe.2015.01.002

Christopher, M., \& Tatham, P. (2011). Humanitarian logistics. Meeting the challenge of preparing for and responding to disasters (pp. 1-14). London: Kogan Page.

Council of supply chain management professionals [CSCMP]. (2011).

Cozzolino, A. (2012). Humanitarian Logistics and Supply Chain Management Humanitarian Logistics. Springer Briefs in Business. http://dx.doi.org/10.1007/978-3-642-30186-5_2

Cozzolino, A., Rossi, S., \& Conforti, A. (2012). Agile and Lean Principles in the humanitarian supply chain. The case of the United Nations world food program. Journal of Humanitarian Logistics and Supply Chain Management, 2(1), 16-33. http://dx.doi.org/10.1108/20426741211225984

Devos, G. G. M., \& Carpinetti, L. C. R. (2011). A fuzzy logic approach to supply chain performance management. 
Dong, W. D., Young, H. L., Sung, H. A., \& Min, K. H. (2011). A framework for measuring the performance of service supply chain management.

Dorit, B. (2013). Performance Measurement in Humanitarian Logistics-a process-oriented perspective. University of Applied Sciences Fulda, Germany.

Duen, Y. Y., Ching, C. H., \& Mei, L. Ch. (2006). A modified two-tuple FLC model for evaluating the performance of SCM: By the Six Sigma DMAIC process.

El-Baz, M. A. (2010). Fuzzy performance measurement of a supply chain in manufacturing companies.

Ergun, O., Karakus, G., Keskinocak, P., Swann, J., \& Villarreal, M. (2009). Humanitarian Supply Chain Management-An Overview.

Erkan, C., Mohammet, G., Nezir, A., Alev, T. G., \& Ali, F. G. (2015). A comprehensive review of multi criteria decision making approaches based on interval type-2 fuzzy sets. Knowledge-Based Systems. http://dx.doi.org/10.1016/j.knosys.2015.06.004

Fan, X., Shujun, Zh. S., Wang, L., Yang, Y., \& Hapeshi, K. (2013). An Evaluation Model of Supply Chain Performances Using 5DBSC and LMBP Neural Network Algorithm. Journal of Bionic Engineering, 10, 383-395. http://dx.doi.org/10.1016/S1672-6529(13)60234-6

Fong, F. W., Chao, T. S. (2007). Performance evaluation of a multi-echelon production, transportation and distribution system: A matrix analytical approach.

Gershenson, C. (2011). Artificial Neural Networks for Beginners. EPFL. Retrieved from http://diwww.epfl.ch/mantra/tutorial/english/

Gholamreza, S. J., Naini, Aliahmadi, A. R., \& Eskandari, M. J., (2010). Designing a mixed performance measurement system for environmental supply chain management using evolutionary game theory and balanced score card: A study of an auto industry supply chain.

Gilberto, M. D. G., \& Luiz, C. R. C. (2011). A fuzzy logic approach to supply chain performance management. International Journal of Production Economics.

Ginap, M. A. (2013). Introduction to SCOR. Supply Chain Council Europe conference. Retrieved from www.supply-chain.org

Golparvara, M., \& Seifbarghya, M. (2009). Application of SCOR Model in an Oil- producing Company. Journal of Industrial Engineering, 4, 59-69.

Gunasekarana, A., Patel, C., \& McGaughey, R. E. (2004). A framework for supply chain performance measurement. International Journal of Production Economics, 87, 333-347. http://dx.doi.org/10.1016/j.ijpe.2003.08.003

Gunasekarana, A., Patelb, C., \& Ronald, E. M. (2003). A framework for supply chain performance measurement

Haavisto, I. (2014). Performance in Humanitarian Supply Chains. Publication of Hanken School of Economics, Helsinki.

Halme, J. S. (2005). Global supply chain management and performance measurement: Literature Review.

Handfield, R. B., \& Nichols, E. L. (2002). Supply chain redesign, transforming supply chains in to integrated value systems. Financial Times Prentice Hall.

Howden, M. (2009). How Humanitarian Logistics Information Systems Can Improve Humanitarian Supply Chains: A View from the Field. In J. Landgren \& S. Jul (eds.), Proceedings of the 6th International ISCRAM Conference, Gothenburg, Sweden.

I-Chiu, C., Hsin, G. H., Hsueh, C. L., Ming, C. H., Sing, L. Ch., \& David, C. Y. (2007). A neural network evaluation model for ERP performance from SCM perspective to enhance enterprise competitive advantage.

Ikhsan, B. B., Kuncoro, H. W., \& Dyah, I. (2015). Evaluation of Poultry Supply Chain Performance in XYZ Slaughtering House Yogyakarta using SCOR and AHP Method. Agriculture and Agricultural Science Procedia.

Ince, H., Imamoglu, S. Z., Keskin, H., \& Akgun, A., Efe, M. N. (2013). The impact of ERP systems and Supply Chain Management Practices on Firm Performance: Case of Turkish Companies. 9th International Strategic Management Conference. http://dx.doi.org/10.1016/j.sbspro.2013.10.586 
Irfan, D., Xiaofei, X., \& Chun, D. S. (2008). A SCOR Reference Model of the Supply Chain Management System in an Enterprise. The International Arab Journal of Information Technology.

Jammernegg, W., \& Reiner, G. (2007). Performance improvement of supply chain processes by coordinated inventory and capacity management.

Jiuping, X., Bin, L., \& Desheng, W. (2009). Rough data envelopment analysis and its application to supply chain performance evaluation.

Kaatrud, D. B., Samii, R., \& van Wassenhove, L. N. (2003). UN joint logistics center: a coordinated response to common humanitarian logistics concerns. Forced Migration Review, 18, 11-14.

Kojima, M., Nakashima, K., \& Ohno, K. (2008). Performance evaluation of SCM in JIT environment.

Kovács, G., \& Spens, K. M. (2007). Humanitarian logistics in disaster relief operations. International Journal of Physical Distribution \& Logistics Management.

Krenker, A., Bešter, J., \& Kos, A. (2011). Introduction to the Artificial Neural Networks. http://dx.doi.org/10.5772/15751

Lai, H. K., Ngai, E. W. T., \& Cheng, T. C. E. (2003). An empirical study of supply chain performance in transport logistics.

Lambert, M. D., \& Pohlen L. T. (2001). Supply Chain Metrics. The International Journal of Logistics Management, 12(1), 1-19. http://dx.doi.org/10.1108/09574090110806190

Lin, R. J. (2011). Using fuzzy DEMATEL to evaluate the green supply chain management practices.

Lockamy, A., \& McCormack, K. (2004). Linking SCOR planning practices to supply chain performance: An exploratory study. International Journal of Operations \& Production Management. http://dx.doi.org/10.1108/01443570410569010

Long, D. (1997). Logistics for disaster relief: Engineering on the run. IIE Solutions, 29(6), 26-29.

Long, D. C., \& Wood, D. F. (1995). The logistics of famine relief. Journal of Business Logistics, 16(1), 213-229.

Mansoornejada, B., Pistikopoulosb, E. N., \& Stuart, P. (2013). Metrics for evaluating the forest bio refinery supply chain performance.

Mentzer, J. T., DeWitt, W., Keebler, J. S., Min, S., Nix, N. W., Smith, C. D., \& Zacharia, Z. G. (2001). Defining Supply Chain Management. Journal of Business Logistics, 22(2), 1-25. http://dx.doi.org/10.1002/j.2158-1592.2001.tb00001.x

Mikalef, P., Pateli, A., Batenburg, R., \& Wetering, R. (2013). Investigating the impact of procurement alignment on supply chain management performance. Procedia Technology, 9, 310-319. http://dx.doi.org/10.1016/j.protcy.2013.12.035

Murray, S. (2005). How to deliver on the promises: supply chain logistics: humanitarian agencies are learning lessons from business in bringing essential supplies to regions hit by the tsunami. Financial Times, p. 9.

Nagurney, A. (2012). Commercial vs. Humanitarian Supply Chains.

Neely, A., Mills, J., Platts, K., Richards, H., Gregory, M., Bourne, M., \& Kennerley, M. (2000). Performance measurement system design: developing and testing a process-based approach. International Journal of Operations \& Production Management, 20(10), 1119-1145. http://dx.doi.org/10.1108/01443570010343708

Oloruntoba, R., \& Gray, R. (2006). Humanitarian aid: an agile supply chain. InternationalJournal of Supply Chain Management, 11(2), 115-120. http://dx.doi.org/10.1108/13598540610652492

Olugu, E. U., \& Wong, K. Y. (2011). An expert fuzzy rule-based system for closed-loop supply chain performance assessment in the automotive industry.

Öztemel, E., (2003). YapayStnırAğları. PapatyaYayıncılık.

Photis, M., Panayides, H. V., \& Lun, Y. (2009). The impact of trust on innovativeness and supply chain performance.

Poister, T. H. (2003). Measuring Performance in Public and Nonprofit Organizations. San Francisco: Jossey-Bass.

Qiang, P., \& Nagurney, A. (2012). A bi-criteria indicator to assess supply chain network performance for critical needs under capacity and demand disruptions. 
Qing, H. Z., Sarkis, J., \& Kee, H. L. (2013). Institutional-based antecedents and performance outcomes of internal and external green supply chain management practices. Journal of Purchasing \& Supply Management.

Raghavendra, T. V. S., Arudhra, N., Amaranath, K., Raviteja, B., \& Sreekar, G. (2014). Humanitarian Supply Chain Model for Flood Relief: A Case Study Analysis. International Journal of Engineering Research \& Technology (IJERT).

Reiner, G. (2004). Customer-oriented improvement and evaluation of supply chain processes supported by simulation models.

Reza, R., Kannan, G., Ahmad, E., \& Mahdi, S. (2015). Application of fuzzy VIKOR for evaluation of green supply chain management practices. Ecological Indicators.

Rodman, W. R. (2004). Supply chain management in humanitarian relief logistics. Master's Thesis, Air Force Institute of Technology Wright-Patterson Air Force Base, Ohio.

Rostamzadeh, R., Govindan, K., Esmaeili, A., \& Sabaghi, M. (2015). Application of fuzzy VIKOR for evaluation of green supply chain management practices.

Ru, J. L., Rong, H. Ch., \& Thi, H. N. (2011). Green supply chain management performance in automobile manufacturing industry under uncertainty.

Sander, D. L. (2014). Towards a reference mission map for performance measurement in humanitarian supply chains.

Santarelli, G., Abidi, H., Regattieri, A., \& Klumpp, M. (2013). A performance measurementsystem for the evaluation of humanitarian supply chains.

Saranga, H., \& Roger, M. (2010). Performance evaluation of purchasing and supply management using value chain DEA approach.

Scholten, K., Scott, P. S., \& Fynes, B. (2010). (Le) agility in humanitarian aid (NGO) supply chains.International Journal of Physical Distribution \& Logistics Management, 40(8/9), 623-635. http://dx.doi.org/10.1108/09600031011079292

Schulz, S. F., \& Heigh, I. (2009). Logistics performance management in action within a humanitarian organization. Management Research News, 32(11), 1038-1049. http://dx.doi.org/10.1108/01409170910998273

Shafiee, M., Lotfi, F. H., \& Saleh, H. (2014). Supply chain performance evaluation with data envelopment analysis and balanced scorecard approach.

Shenc, L., Olfat, L., Govindan, K., Khodaverdi, R., \& Diabat, A. (2012). A fuzzy multi criteria approach for evaluating green supplier's performance in green supply chain with linguistic preferences.

Shepherd, C., \& Gunter, H. (2006). Measuring supply chain performance: current re-search and future directions. International Journal of Productivity and Performance Management, 55(3/4), 242-258. http://dx.doi.org/10.1108/17410400610653219

Sukati, I., Hamid, A. B., Baharun, R., \&Yusoff, R. M. (2012). The study of supply chain management strategy and practices on supply chain performance. Procedia: social and behavioral sciences, 40, 225-233. http://dx.doi.org/10.1016/j.sbspro.2012.03.185

Supply Chain Council. (2010). Supply Chain Operations Reference Model: Version 10. Retrieved from www.supply-chain.org

Thomas, A., \& Kopczak, L. R. (2005). From Logistics to Supply Chain Management: The path forward in the Humanitarian sector. Fritz Institute.

Trkman, P., McCormack, K., Valadares, de O. M. P., \& Marcelo, B. L. M. B. (2010). The impact of business analytics on supply chain performance.

Tsoulfas, G. T., \& Pappis, C. P. (2008). A model for supply chains environmental performance analysis and decision making.

Vahrenkamp, R., \& Siepermann, C. (2005). Logistics: Management and Strategies. München: OldenbourgVerlag.

Vidalis, M. I., Koukoumialos, B., \& Michael, G. (2013). Performance evaluation of a merge supply network: A distribution centre with multiple reliable random suppliers.

Wassenhove, L. V. (2006). Humanitarian aid logistics: supply chain management in highgear. Journal of the Operational Research Society, 57, 475-489. http://dx.doi.org/10.1057/palgrave.jors.2602125 
Willner, D., Zafeiridis, S., \& Waugh, B. (2013). Challenges and the use of performance measurements in humanitarian supply chains. Master's Thesis, Jönköping University, Business Administration.

Wong, T. C., Law, K. M. Y., \& Ngan, S. C. (2010). Analyzing supply chain operation models with the $\mathrm{PC}$-algorithm and the neural network.

Yeong, D. H., Yi, C. L., \& Lyu, J. Jr. (2008). The performance evaluation of SCOR sourcing process-the case study of Taiwan's TFT-LCD industry.

Youn, S., Yang, M. G., Hong, P., \& Park, K. (2013). Strategic supply chain partnership, environmental supply chain management practices and performance outcomes: an empirical study of Korean firms. Journal of cleaner production, 56, 121-130. http://dx.doi.org/10.1016/j.jclepro.2011.09.026

Zadeh, L. A. (1965). Fuzzy sets. Information and Control, 8(3), 338-353.

Zafeiridis, D. W. S. (2013). Challenges and the use of performance measurements in humanitarian supply chains.

\section{Copyrights}

Copyright for this article is retained by the author, with first publication rights granted to the journal.

This is an open-access article distributed under the terms and conditions of the Creative Commons Attribution license (http://creativecommons.org/licenses/by/3.0/) 